\title{
Effect of Repetitive Transcranial Magnetic Stimulation on Patients With Dysarthria After Subacute Stroke
}

\author{
Yong Gyu Kwon, $\mathrm{MD}^{1}$, Kyung Hee Do, MD ${ }^{1}$, Sung Jong Park ${ }^{1}$, \\ Min Cheol Chang, $\mathrm{MD}^{2}$, Min Ho Chun, MD, $\mathrm{PhD}^{1}$ \\ ${ }^{1}$ Department of Rehabilitation Medicine, Asan Medical Center, University of Ulsan College of Medicine, Seoul; \\ ${ }^{2}$ Rehabilitation Center, Union Hospital, Daegu, Korea
}

Objective To evaluate whether repetitive transcranial magnetic stimulation (rTMS) could improve dysarthria in stroke patients at the subacute stage.

Methods This study was a prospective, randomized, double-blind controlled trial. Patients who had unilateral middle cerebral artery infarction were enrolled. In patients in the rTMS group, we found hot spots by searching for the evoked motor potential of the orbicularis oris on the non-affected side. We performed rTMS at a low frequency $(1 \mathrm{~Hz}), 1,500$ stimulations/day, 5 days a week for 2 weeks on the hotspots. We used the same protocol in the sham stimulation group patients as that in the rTMS group, except that the angle of the coil was perpendicular to the skull rather than tangential to it. The patients in both groups received speech therapy for 30 minutes, 5 days a week from a skilled speech therapist. The speech therapist measured the Urimal Test of Articulation and Phonology, alternative motion rates, sequential motion rates, and maximal phonation time before and after intervention sessions.

Results Forty-two patients were enrolled in this study and 20 completed the study. Statistical analysis revealed significant improvements on the dysarthria scales in both groups. The sequential motion rate (SMR)-PəТəKə showed significantly greater improvement in the rTMS group patients than in the sham stimulation group.

Conclusion Patients in the rTMS group showed greater improvement in articulation than did patients in the sham rTMS group. Therefore, rTMS can have a synergistic effect with speech therapy in treating dysarthria after stroke.

Keywords Dysarthria, Stroke, Repetitive transcranial magnetic stimulation, Orbicularis oris

Received May 11, 2015; Accepted June 3, 2015

Corresponding author: Min Ho Chun

Department of Rehabilitation Medicine, Asan Medical Center, University of Ulsan College of Medicine, 88 Olympic-ro 43-gil, Songpa-gu, Seoul 05505, Korea

Tel: +82-2-3010-3796, Fax: +82-2-3010-6964, E-mail: mhchun@amc. seoul.kr

(c) This is an open-access article distributed under the terms of the Creative Commons Attribution Non-Commercial License (http://creativecommons. org/licenses/by-nc/4.0) which permits unrestricted noncommercial use, distribution, and reproduction in any medium, provided the original work is properly cited.

Copyright $\odot 2015$ by Korean Academy of Rehabilitation Medicine

\section{INTRODUCTION}

Dysarthria is defined as a neurologic motor speech impairment characterized by slow, weak, imprecise, and/ or uncoordinated movements of the speech musculature, and it may involve respiration, phonation, resonance, and/or oral articulation [1]. Stroke may result in weakness, paralysis, or a lack of coordination of the motorspeech system, producing dysarthria [2]. Flowers et al. 
[3] reported an incidence of dysarthria after stroke of $42 \%$ (95\% confidence interval [CI], 35\%-48\%) and found that the highest predictor was weakness (odds ratio [OR], 5.3; CI, 2.4-12.0). Brady et al. [4] reported that dysarthria transcended physiological impairment to impact social participation. However, there have been few studies on the effect of speech therapy on dysarthria after stroke. Sellars et al. [5] reported that only small-size clinical trials had shown that speech therapy could help to improve dysarthria after stroke. There have also been no previous studies of treatments for dysarthria other than speech therapy.

Repetitive transcranial magnetic stimulation (rTMS) may be an alternative therapy for dysarthria in addition to conventional speech therapy. rTMS is a noninvasive procedure that uses magnetic fields to create electric currents in discrete brain areas [6-11]. Many studies have reported that rTMS helps to improve limb weakness after stroke and that low-frequency (LF) pulses are particularly more effective than those of high frequency (HF) [12]. Considering that dysarthria is caused by weakness in the muscles that enable articulation, we had a strong expectation that rTMS would help to improve the symptoms of dysarthria after stroke. To address this issue, we performed randomized and sham stimulation-controlled studies of patients with subacute stroke to investigate this possibility and describe the clinical outcomes of this therapy.

\section{MATERIALS AND METHODS}

\section{Subjects}

This study was a single-center, prospective, randomized, double-blind, sham stimulation-controlled trial. Stroke patients who were treated from June 2013 to April 2015 in the Department of Rehabilitation Medicine at Asan Medical Center were enrolled. All study subjects were screened for cognitive dysfunction using the Korean version of the Mini-Mental Status Examination (MMSE). Our study comprised patients who had experienced a first-ever unilateral middle cerebral artery infarction. The duration from stroke onset ranged from 1 week to 2 months, but all of the study patients had experienced their first-ever stroke. A skillful speech therapist evaluated cognitive function and speech function using evaluation tools such as the Korean version of the Western
Aphasia Battery, and patients who had aphasia, apraxia of speech, cognitive impairment (MMSE $<20$ ), poor mental status, vocal cord palsy, history of epilepsy, or bilateral infarction were excluded. We estimated the target number of participants with reference to a previous study [13]. All participants provided written informed consent, and our local Ethics Committee approved the study protocol.

\section{Motor evoked potentials}

To determine the resting motor threshold and stimulation area, we recorded motor-evoked potentials from the orbicularis oris muscles on each patient's non-affected side using transcranial magnetic stimulation. Focal transcranial magnetic stimulation was applied using a Magstim Rapid magnetic stimulator (Magstim Company Ltd., Dyfed, UK). Briefly, a Magstim circular coil (external diameter, $90 \mathrm{~mm}$ ) was placed onto each subject's contralateral motor cortex to identify the hotspot, defined as the area that produced the largest amplitude of motor-evoked potentials. The resting motor threshold was defined as the stimulus intensity required to produce motor-evoked potentials $>100 \mathrm{kV}$ at a peak-to-peak amplitude during three of five consecutive trials on the orbicularis oris.

\section{Interventions}

We randomly sorted our enrolled stroke patients into two groups: an rTMS group and a sham stimulation group. Randomization was done according to a table of random numbers; odd numbers went to the rTMS group and even numbers went to the sham stimulation group. According to previous studies, LF rTMS on the non-affected side is more effective in producing recovery from weakness than is HF rTMS on the affected side [12], and we thus decided to use only LF rTMS. While seated in a comfortable chair with foam ear plugs, each patient was treated with 10 consecutive sessions (five times per week for 2 weeks) of rTMS, performed by a physiatrist who used a 70-mm, aircooled, figure-of-eight $\mathrm{Y}$-shaped coil.

We performed rTMS at a low frequency $(1 \mathrm{~Hz})$, at $90 \%$ amplitude of evoked motor threshold, and with 1,500 stimulations/day on the hotspot. We applied the same type of LF protocol that was described in the study by Takeuchi et al. [14]. The sham stimulation occurred using the same protocol as that for the LF stimulation, except that the angle of the coil was perpendicular to the skull 
rather that tangential to it. Thus, the magnetic field could not penetrate the brain, although the subjects could hear the sound that was produced. This protocol has been used in other studies [14-17]. The subjects in both study groups received speech therapy for 30 minutes, 5 days a week from a skilled speech therapist who was blind to the nature of the study during the 2-week intervention period. The patients were also unaware of which group they had been assigned to.

\section{Evaluation}

Dysarthria was evaluated by a single skilled speech therapist who was blind to the study protocol. This clinician performed the Urimal Test of Articulation and Phonology (U-TAP), and measured alternative motion rates (AMR), sequential motion rates (SMR), and the maximal phonation time (MPT) as outcome measures before and after the rTMS sessions. These tests were verified for evaluating dysarthria in a number of studies and they were designed to measure the velocity of movement and coordination of articulators including the tongue and lips [13]. MPT was determined by measuring the duration of the /a/ vowel produced at a comfortable amplitude and pitch level after maximum inspiration. Following appropriate instructions, this procedure was repeated three times by the subject, and the recording with the longest duration was considered to be the MPT. Subjects were then asked to repeat the phonemes /pə/, /tə/, and $/ \mathrm{k}$ / as rapidly as possible and for as long as possible at a comfortable pitch and loudness. This test is used for the clinical assessment of speech motor function [18]. Diadochokinesis (DDK) can be used to measure syllable timing and rhythm in speech [19]. Irregular syllable timing during DDK is strongly associated with ataxic dysarthria and can be associated with spastic dysarthria as well [20]. DDK measures are divided into two types: AMR and SMR. AMR is measured as the rapid repetition of a single syllable /pə/ and SMR is measured as the rapid repetition of a sequence of syllables (/pə/, /tə/, and / kə/).

We evaluated sex, age, duration from stroke, and location of the stroke as the baseline characteristics of our study patients. We also evaluated their Modified Barthel Index (MBI) and Motricity index scores before beginning the rTMS sessions.

\section{Statistical analysis}

Statistical analyses were performed with SPSS ver. 18 (SPSS Inc., Chicago, IL, USA). Data are presented as mean \pm standard deviation. The general characteristics of the two study groups were analyzed with the MannWhitney U test and Fisher exact test. Paired comparisons within the groups (before and immediately after rTMS) were analyzed using the Wilcoxon signed-rank test. A pvalue $\leq 0.05$ was considered to indicate statistical significance. Comparisons of interval changes between the two groups were analyzed with the Mann-Whitney U test.

\section{RESULTS}

A total of 42 patients were initially enrolled in this study, but 17 were excluded after being assessed for eligibility. Among the excluded patients, 11 did not meet the inclusion criteria and six refused to participate. A final total of 25 post-stroke patients were therefore recruited and randomized into the two study groups. Twenty of these patients completed the study. Three and two patients were unable to complete the study in the rTMS and sham stimulation groups, respectively (Fig. 1). Ten patients each completed the rTMS and sham stimulation groups. No major side effects of rTMS administration, such as seizures or headaches, were reported for any patient who completed the 10 consecutive sessions.

The baseline characteristics of the rTMS and sham stimulation patients were not significantly different (Table 1). The mean age of the subjects was 69.4 years in the rTMS group vs. 68.8 years in the sham stimulation group. The average duration after stroke was 26.4 days in the rTMS group vs. 26.5 days in the sham stimulation group, and there was no significant difference in the location of the stroke. Between these two groups, there were also no significant differences in terms of MBI or Motricity index scores. Table 2 lists the outcome parameters of the two groups after treatment. Wilcoxon signed-rank test analysis showed that all outcome measures were significantly improved after rTMS and conventional speech therapy. The sham stimulation patients also showed improvement in their AMR-Pə, AMR-Tə, AMR-Kə, and MPT scores and displayed a tendency toward improved U-TAP scores.

Table 3 lists a comparison of parameter differences after sham stimulation and rTMS. The rTMS group patients showed significantly greater improvement in the 


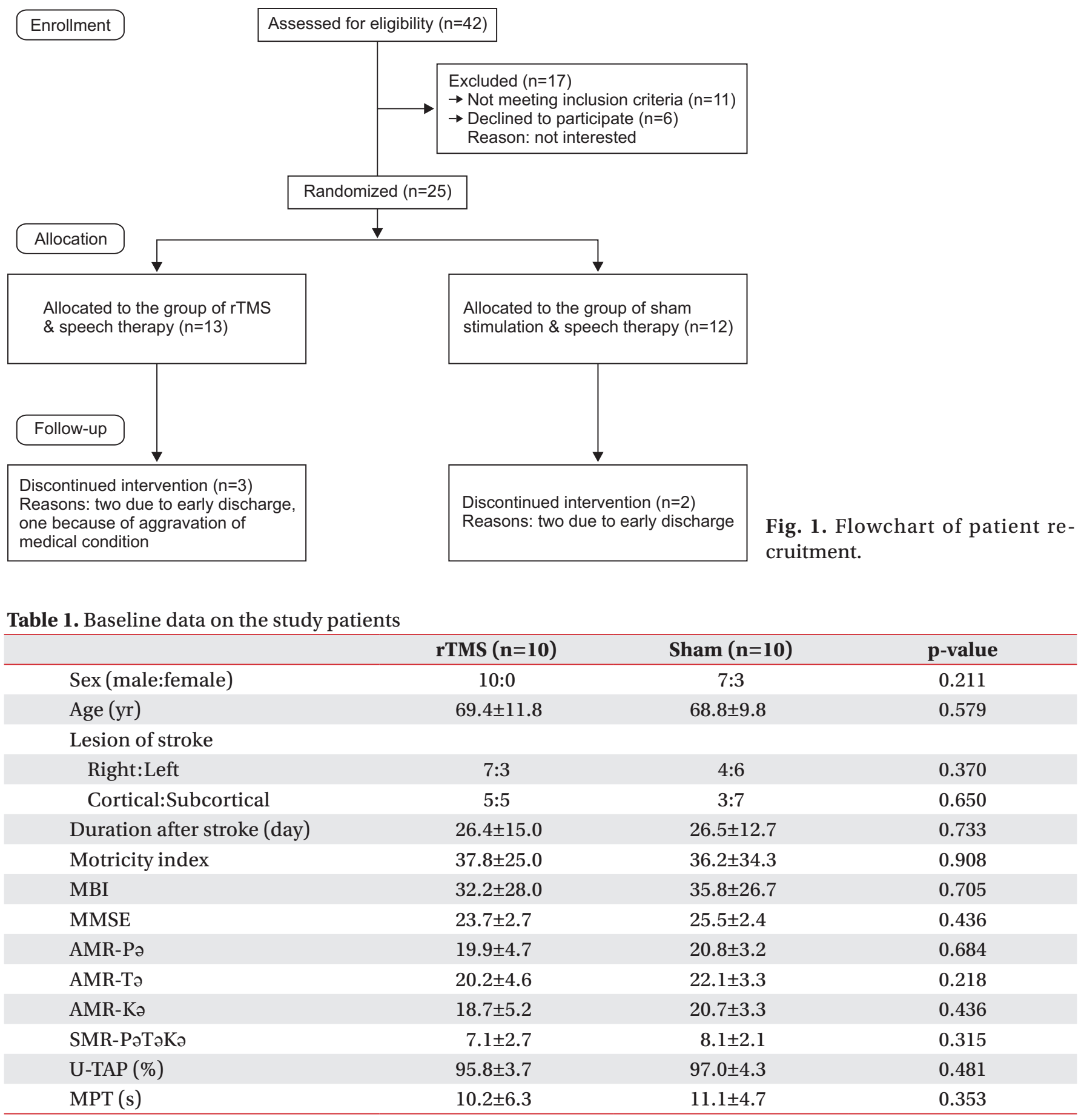

Values are presented as mean \pm standard deviation or number.

rTMS, repetitive transcranial magnetic stimulation; AMR, alternative motion rate; MPT, maximal phonation time; MBI, Modified Barthel Index; MMSE, Mini-Mental Status Examination; U-TAP, Urimal Test of Articulation and Phonology.

${ }^{*} \mathrm{p}<0.05$ by Mann-Whitney U test and Fisher exact test.

SMR-PəTəKə score than did the sham stimulation group. The AMR-Pə and U-TAP scores also showed a tendency towards greater improvement in the rTMS group than in the sham stimulation group, but this difference was not found to be significant. 
Table 2. Comparison of the differences before and after treatment in the rTMS and sham stimulation groups

\begin{tabular}{|c|c|c|c|c|c|c|}
\hline & \multicolumn{2}{|c|}{ rTMS $(n=10)$} & \multirow{2}{*}{ p-value } & \multicolumn{2}{|c|}{ Sham $(n=10)$} & \multirow{2}{*}{ p-value } \\
\hline & Before & After & & Before & After & \\
\hline AMR-Pə & $19.9 \pm 4.7$ & $23.0 \pm 4.4$ & $0.005^{*}$ & $20.8 \pm 3.2$ & $22.5 \pm 3.0$ & $0.038^{*}$ \\
\hline AMR-Tə & $20.2 \pm 4.6$ & $22.9 \pm 4.5$ & $0.040^{*}$ & $22.1 \pm 3.3$ & $23.7 \pm 2.5$ & $0.027^{*}$ \\
\hline AMR-Kə & $18.7 \pm 5.2$ & $22.2 \pm 5.5$ & $0.012^{*}$ & $20.7 \pm 3.2$ & $22.2 \pm 4.1$ & $0.027^{*}$ \\
\hline AMR-РəТəКə & $7.1 \pm 2.7$ & $9.9 \pm 3.8$ & $0.011^{*}$ & $8.1 \pm 2.1$ & $8.5 \pm 2.1$ & 0.157 \\
\hline U-TAP & $95.8 \pm 3.7$ & $100.0 \pm 0.0$ & $0.011^{*}$ & $96.7 \pm 4.3$ & $98.1 \pm 4.7$ & 0.059 \\
\hline MPT & $10.2 \pm 6.3$ & $12.4 \pm 4.5$ & $0.011^{*}$ & $11.1 \pm 4.7$ & $13.2 \pm 3.6$ & $0.007^{*}$ \\
\hline
\end{tabular}

Values are presented as mean \pm standard deviation.

rTMS, repetitive transcranial magnetic stimulation; AMR, alternative motion rate; MPT, maximal phonation time; UTAP, Urimal Test of Articulation and Phonology.

${ }^{*} \mathrm{p}<0.05$ by Wilcoxon signed-rank test.

Table 3. Differences between the rTMS $(n=10)$ and sham stimulation $(n=10)$ groups in terms of the speech parameters indicated

\begin{tabular}{lccc}
\hline & rTMS (n=10) & Sham $(\mathbf{n}=\mathbf{1 0})$ & p-value \\
\hline$\triangle$ AMR-Pə & $3.1 \pm 2.3$ & $1.3 \pm 1.5$ & 0.064 \\
$\triangle$ AMR-Tə & $2.7 \pm 3.8$ & $1.6 \pm 1.6$ & 0.684 \\
$\Delta$ AMR-Кə & $3.5 \pm 3.1$ & $1.3 \pm 2.0$ & 0.143 \\
$\Delta$ SMR-PəTəКə & $2.8 \pm 3.9$ & $0.4 \pm 0.8$ & $0.043^{*}$ \\
$\Delta$ U-TAP & $4.2 \pm 3.7$ & $1.5 \pm 1.9$ & 0.052 \\
$\Delta$ MPT & $2.2 \pm 3.7$ & $3.3 \pm 2.7$ & 1.000 \\
\hline
\end{tabular}

Values are presented as mean \pm standard deviation. rTMS, repetitive transcranial magnetic stimulation; AMR, alternative motion rate; MPT, maximal phonation time; U-TAP, Urimal Test of Articulation and Phonology. ${ }^{*} \mathrm{p}<0.05$ by the Mann-Whitney $\mathrm{U}$ test.

\section{DISCUSSION}

The primary objective of our present study was to evaluate the effect of rTMS in patients with dysarthria following a subacute stroke. In our analyses, the patients in both the rTMS and sham stimulation groups showed improvement in dysarthria, and the rTMS group showed greater improvement in the SMR-PəTəKə score and a tendency toward improved U-TAP and AMR-Pə scores. We assume from this that speech therapy and natural recovery led to improvements in both of our stroke patient study groups and that rTMS may have contributed to the degree of improvement in some of the tests that were administered.

Recently, many post-stroke treatment options including noninvasive TMS have been suggested as potential new strategies in neurorehabilitative settings $[9,11,14$ 16]. Various patterns of neural reorganization occur after a stroke, and functional recovery is associated with neuroplastic changes in the brain. rTMS can improve motor functions after a stroke, and, according to the interhemispheric competition model, HF stimulation can be administered to the primary motor cortex of the ipsilesional cerebral hemisphere to up-regulate excitability or LF stimulation can be administered to the primary motor cortex of the contralesional cerebral hemisphere to down-regulate it [12]. We surmised from our current study findings that the benefit of rTMS to the muscles required for articulation could be similar to that for limb muscles because dysarthria after a stroke occurs because of the weakness of the tongue, pharyngeal, and laryngeal muscles that are involved in speech articulation [21]. From among these muscles, we found a motor-evoked potential from the orbicularis oris because this muscle was the easiest articulatory muscle to apply the surface electrode to.

The function of the orbicularis oris is to close and compress the lips [22]. During articulation, the muscle helps the buccinator and risorius muscles to produce the /f, v/ phonemes, creates lip compression to produce the phonemes /p, b, m/, and rounds the lips to produce /w/ [23]. This muscle works with the levator labii superioris, mentalis, and levator labii superioris to round and protrude the lips [23]. In terms of the evaluations we conducted in our current study, this muscle could help to achieve improvements in AMR-Pə, SMR-PəTəKə, and U-TAP. Our study findings were consistent with this to some degree, i.e., the SMR-PəТəKə improved more significantly, and 
the U-TAP and AMR Pə showed a tendency toward greater improvement in the rTMS-treated patients than in the sham-stimulated patients.

The difference between our present findings and those of other reports that covered the effect of rTMS on limb muscle recovery is that dysarthria mostly affects the muscles from not only the corticospinal tract but also the corticolingual and facial corticobulbar tracts [24]. Compared with the corticospinal tracts, in which most parts of the fibers innervate the contralateral side, corticolingual fibers are known to project bilaterally from either hemisphere to the hypoglossal nuclei, and the corticoorofacial fibers project predominantly to the contralateral subnuclei [25].

Although the orbicularis oris is mainly innervated by the contralateral hemisphere through the facial nerve, its natural recovery from dysarthria could be better than that of limb weakness because the loss of function in the lingual, velopharyngeal, laryngeal, and respiratory muscles that participate in articulation can be compensated for by fibers from the non-affected side because of the bilateral innervation of the corticolingual and corticobulbar tracts [25].

To our knowledge, this is the first well-controlled, randomized, double-blind study that has applied rTMS to treat dysarthria after stroke. Dysarthria has not been well studied to date because the symptoms of the condition are difficult to measure, and doctors have concentrated mainly on the limb weakness in stroke cases, regarding dysarthria as a minor problem. However, considering the negative psychosocial impact on the lives of these patients after they return home, we believe that this issue should be of greater concern and receive more attention. We have found from our current analysis that rTMS is a possible treatment option for dysarthria and warrants further investigation in large cohorts of stroke patients in the future. It will be interesting to investigate whether there is a difference in the outcomes of HF and LF rTMS between cortical and subcortical lesions and between right- and left-sided lesions. Additional studies that investigate the effect of rTMS for dysarthria should consider the issues that we have raised in our current report.

The main limitations of this study were the relatively small numbers of subjects and the absence of a HF stimulation group involving the affected side. In addition, we did not check the severity of facial palsy, other bulbar symptoms such as dysphagia, or the improvement of other motor functions including the Motricity index and MBI values. If we had evaluated these parameters, the extent of natural recovery in our patients could likely have been more completely understood and excluded.

In conclusion, dysarthria symptoms are improved by rTMS, which confers an additional benefit along with speech therapy in treating dysarthria after stroke. To elucidate the exact effects of this therapy and to determine the most effective application of rTMS for dysarthria, larger-scale studies that apply it in a variety of ways are needed.

\section{CONFLICT OF INTEREST}

No potential conflict of interest relevant to this article was reported.

\section{REFERENCES}

1. Yorkston KM, Beukelman DR. Ataxic dysarthria: treatment sequences based on intelligibility and prosodic considerations. J Speech Hear Disord 1981;46:398404.

2. Martin BJ, Corlew MM. The incidence of communication disorders in dysphagic patients. J Speech Hear Disord 1990;55:28-32.

3. Flowers HL, Silver FL, Fang J, Rochon E, Martino R. The incidence, co-occurrence, and predictors of dysphagia, dysarthria, and aphasia after first-ever acute ischemic stroke. J Commun Disord 2013;46:238-48.

4. Brady MC, Clark AM, Dickson S, Paton G, Barbour RS. The impact of stroke-related dysarthria on social participation and implications for rehabilitation. Disabil Rehabil 2011;33:178-86.

5. Sellars C, Hughes T, Langhorne P. Speech and language therapy for dysarthria due to non-progressive brain damage (review). Chichester: John Wiley \& Sons; 2009.

6. Alonso-Alonso M, Fregni F, Pascual-Leone A. Brain stimulation in poststroke rehabilitation. Cerebrovasc Dis 2007;24 Suppl 1:157-66.

7. Lefaucheur JP. Stroke recovery can be enhanced by using repetitive transcranial magnetic stimulation (rTMS). Neurophysiol Clin 2006;36:105-15.

8. Pascual-Leone A, Amedi A, Fregni F, Merabet LB. The 
plastic human brain cortex. Annu Rev Neurosci 2005; 28:377-401.

9. Peinemann A, Reimer B, Loer C, Quartarone A, Munchau A, Conrad B, et al. Long-lasting increase in corticospinal excitability after 1800 pulses of subthreshold $5 \mathrm{~Hz}$ repetitive TMS to the primary motor cortex. Clin Neurophysiol 2004;115:1519-26.

10. Maeda F, Keenan JP, Tormos JM, Topka H, PascualLeone A. Modulation of corticospinal excitability by repetitive transcranial magnetic stimulation. Clin Neurophysiol 2000;111:800-5.

11. Muellbacher W, Ziemann U, Boroojerdi B, Hallett M. Effects of low-frequency transcranial magnetic stimulation on motor excitability and basic motor behavior. Clin Neurophysiol 2000;111:1002-7.

12. Hsu WY, Cheng CH, Liao KK, Lee IH, Lin YY. Effects of repetitive transcranial magnetic stimulation on motor functions in patients with stroke: a meta-analysis. Stroke 2012;43:1849-57.

13. You DS, Chun MH, Kim DY, Han EY, Jung SE. The effects of transcranial direct current stimulation on dysarthria in stroke patients. J Korean Acad Rehabil Med 2010;34:10-4.

14. Takeuchi N, Chuma T, Matsuo Y, Watanabe I, Ikoma K. Repetitive transcranial magnetic stimulation of contralesional primary motor cortex improves hand function after stroke. Stroke 2005;36:2681-6.

15. Kim YH, You SH, Ko MH, Park JW, Lee KH, Jang SH, et al. Repetitive transcranial magnetic stimulationinduced corticomotor excitability and associated motor skill acquisition in chronic stroke. Stroke 2006;37: 1471-6.

16. Khedr EM, Etraby AE, Hemeda M, Nasef AM, Razek AA. Long-term effect of repetitive transcranial magnetic stimulation on motor function recovery after acute ischemic stroke. Acta Neurol Scand 2010;121:30-7.
17. Talelli P, Greenwood RJ, Rothwell JC. Exploring Theta Burst Stimulation as an intervention to improve motor recovery in chronic stroke. Clin Neurophysiol 2007; 118:333-42.

18. Fimbel EJ, Domingo PP, Lamoureux D, Beuter A. Automatic detection of movement disorders using recordings of rapid alternating movements. J Neurosci Methods 2005;146:183-90.

19. Kent RD, Weismer G, Kent JF, Vorperian HK, Duffy JR. Acoustic studies of dysarthric speech: methods, progress, and potential. J Commun Disord 1999;32:141-80, 183-6.

20. Tjaden K, Watling E. Characteristics of diadochokinesis in multiple sclerosis and Parkinson's disease. Folia Phoniatr Logop 2003;55:241-59.

21. Urban PP, Rolke R, Wicht S, Keilmann A, Stoeter P, Hopf HC, et al. Left-hemispheric dominance for articulation: a prospective study on acute ischaemic dysarthria at different localizations. Brain 2006;129(Pt 3):767-77.

22. Seikel JA, King DW, Drumright DG. Anatomy \& physiology for speech, language, and hearing. 2nd ed. San Diego: Singular Publishing; 2000.

23. Borden GJ, Harris KS, Raphael LJ. Speech science primer: physiology, acoustics, and perception of speech. 3rd ed. Baltimore: Lippincott Williams \& Wilkins; 1994.

24. Liegeois FJ, Morgan AT. Neural bases of childhood speech disorders: lateralization and plasticity for speech functions during development. Neurosci Biobehav Rev 2012;36:439-58.

25. Urban PP, Hopf HC, Fleischer S, Zorowka PG, MullerForell W. Impaired cortico-bulbar tract function in dysarthria due to hemispheric stroke: functional testing using transcranial magnetic stimulation. Brain 1997;120(Pt 6):1077-84. 